\title{
Human Spine Posture Estimation Method from Human Images to Calculate Physical Forces Working on Vertebrae
}

\author{
Daisuke Furukawa ${ }^{1}$, Takayuki Kitasaka ${ }^{2}$ Kensaku Mori ${ }^{2}$, Yasuhito Suenaga ${ }^{2}$, \\ Kenji Mase ${ }^{3}$, and Tomoichi Takahashi ${ }^{4}$ \\ 1 Graduate School of Engineering, Nagoya University \\ Furo-cho, Chikusa-ku, Nagoya, Aichi, 464-8603 Japan \\ 2 Graduate School of Information Science, Nagoya University \\ Furo-cho, Chikusa-ku, Nagoya, Aichi, 464-8603 Japan \\ 3 Information Technology Center, Nagoya University \\ Furo-cho, Chikusa-ku, Nagoya, Aichi, 464-8603 Japan \\ 4 Faculty of Science and Technology, Meijo University \\ Shiogamaguchi, Tempaku-ku, Nagoya, Aichi, 468-8502 Japan
}

\begin{abstract}
This paper describes a method for estimating a human spine posture from human images using a human spine model to compute the rough approximation of the physical forces working on vertebral bodies. Our method uses the positions of the neck and waist in addition to the positions of the head, torso, and arms estimated from the actual human images. The spine model constructed from 3-D CT images is deformed to place the top and the bottom vertebrae of the spine model to the estimated neck and waist positions. According to the experimental results based on one real MR image dataset of one subject person, our methods estimated the positions of the vertebrae within positional shifts of about $6.3 \mathrm{~mm}$ and the rotational variation of about 3.1 degrees. We also confirmed the methods calculated the reasonable estimation of the physical forces working on the vertebral body.
\end{abstract}

\section{Introduction}

Recently, there is a growing concern with the physical load analysis of the spine, since diseases such as low back pain due to damage to the spine frequently occur to us in our daily life 123 . In these studies, local spine models composed of some vertebrae and surrounding muscles are used for numerical computation. Initial conditions and boundary conditions are adjusted to satisfy the situation that we have to analyze such as a subject's posture, external forces, and so on. Under those conditions, internal stress inside the vertebral bodies are calculated using the finite element method. Generally, those conditions are determined manually, hence analyzed posture should be restricted to a simple one.

If we can determine the initial conditions from human images automatically, we can easily compute internal stress for a real posture in which a subject 
actually holds. To determine the initial conditions, we have to estimate the orientations of the vertebrae and the physical forces working on the vertebrae from human images.

There are few studies on the method that is able to fit a spine model to an actual human image, and to estimate pressure on the vertebrae simultaneously. Some researches on either posture generation or model fitting using a human body model are reported. Badler et al. 4. proposed spine and torso models and a motion generation method using them for computer animation. Otake et al. [5] overlaid a skeletal model of the lower half of the body onto human images using motion capture data. However, it is difficult to apply these methods to physical force computation, since the models are deformed based on kinematics.

We proposed a method for estimating the positions and the orientations of the vertebrae from human images [6]. In this method, the spine model is deformed to place the top and bottom vertebrae on the neck and waist positions obtained from input human images by applying two virtual forces to the top and bottom vertebrae corresponding to the neck and waist. Even though virtual forces were used in our previous method because of the difficulty of obtaining the physically appropriate forces, we were able to estimate the spine postures reasonably.

This paper describes a method for computing the rough approximation of the physical forces working on vertebral bodies as well as for estimating the positions and the orientations of the vertebrae. In Sect.2, we present the spine posture estimation model. The spine posture estimation process is described in Sect. 3 The physical force computation is also explained briefly. In Sect. 4 we show the experimental results of evaluation of the accuracy of the estimated positions and the orientations of the vertebrae. Then we show the preliminary experimental results of the physical force computation. Section 5 contains conclusions and future works.

\section{Spine Posture Estimation Model}

The human spine is mainly composed of vertebrae and intervertebral discs. We model the vertebrae and the intervertebral discs as rigid body and elastic body, respectively (Fig. 1). Each vertebra is represented as a surface object since the vertebrae are dealt with as rigid bodies in the model deformation process. An intervertebral disk is modeled as eight springs connecting the adjacent vertebrae. Movement of the vertebrae in the deformation process is constrained by the springs.

A set of X-ray CT images provided by the Visible Human Project [7] is used to construct the model. The surface of the vertebral bodies are determined by applying the marching cubes method to the voxel data obtained by simply thresholding the CT images. The contacting points of the vertebra and the associated springs are manually adjusted considering the center of mass of the vertebra and the symmetry of the arrangement of the springs. 


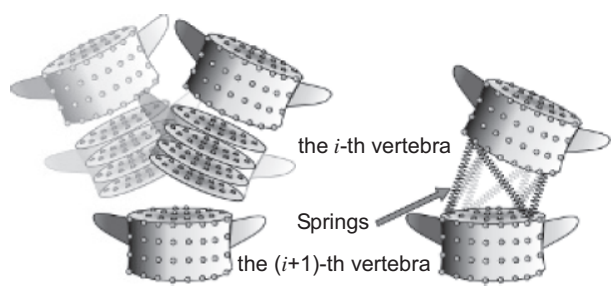

Fig. 1. Spine posture estimation model.

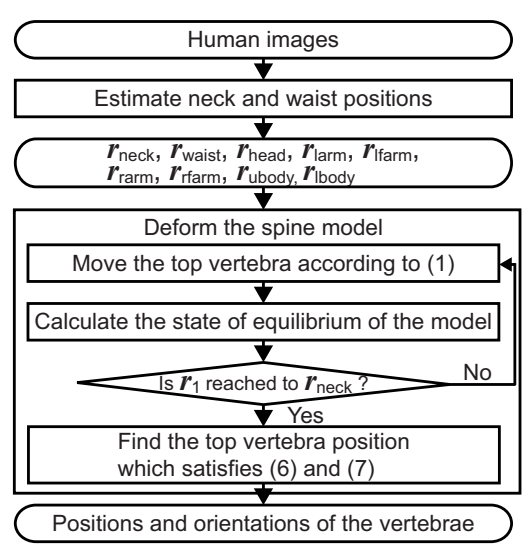

Fig. 2. Processing flow of the spine posture estimation.

\section{Spine Posture Estimation Method}

\subsection{Neck and Waist Positions Estimation}

Figure 2 shows the processing flow of the spine posture estimation method. The input is a sequence of human images of a subject taken by video cameras.

We use the whole body model to estimate the neck and waist positions, and the centers of mass of the head, arms, and upper and lower bodies from the input images. The whole body model consists of eleven parts of ellipsoids that represent the head, upper and lower bodies, upper arms and forearms, and thighs and legs. Although the whole body model was manually registered to the human images in this paper, conventional posture estimation methods such as 10 can be used for registration. After the model fitting, the neck position $\boldsymbol{r}_{\text {neck }}$ and the waist position $\boldsymbol{r}_{\text {waist }}$ are obtained by the end point of the ellipsoid corresponding to the upper body, and the center of mass of the ellipsoid corresponding to the lower body. The centers of mass of the head, arms, and bodies, $\boldsymbol{r}_{\text {head }}, \boldsymbol{r}_{\text {larm }}$, $\boldsymbol{r}_{\text {lfarm }}, \boldsymbol{r}_{\text {rarm }}, \boldsymbol{r}_{\text {rfarm }}, \boldsymbol{r}_{\text {ubody }}$, and $\boldsymbol{r}_{\text {lbody }}$ are approximated by the centers of mass of the corresponding ellipsoids.

\subsection{Spine Model Deformation}

First, the spine model is translated and rotated to make the position and the orientation of the bottom vertebra of the spine model coincide with those of the waist estimated from the inputs. Then the top vertebra is moved toward the neck position by a small distance. This movement is carried out iteratively until the top vertebra reaches $\boldsymbol{r}_{\text {neck }}$. After $n$ iterations, the top vertebra position $\boldsymbol{r}_{1}(n)$ is expressed by

$$
\boldsymbol{r}_{1}(n)=\boldsymbol{r}_{1}(n-1)+\delta \boldsymbol{d},
$$


where $\boldsymbol{d}$ satisfies $\boldsymbol{d}=\boldsymbol{r}_{\text {neck }}-\boldsymbol{r}_{1}(0)$, and $\delta \boldsymbol{d}$ means the small displacement along the direction of $\boldsymbol{d} . \boldsymbol{r}_{1}(0)$ is the initial position before the model deformation process.

Each time the top vertebra is moved by $\delta \boldsymbol{d}$, the positions and the orientations of the vertebrae except the top and bottom vertebrae are calculated so that the spine model satisfies equilibrium. We consider two types of forces working on the $i$-th vertebra. The first type is the weight $\boldsymbol{W}_{i}$ of the body supported by the $i$-th vertebra. To calculate $\boldsymbol{W}_{i}$, we use the information about the positions and the orientations of the ellipsoids that approximate the torso in the whole body model. The weight $\boldsymbol{W}_{i}$ can be calculated by integrating the volume defined by the surfaces of the ellipsoids and the planes passing through the centers of mass of the $(i-1)$-th and the $i$-th vertebrae. The second one is the forces of repulsion $\boldsymbol{T}_{i j}$ of the springs connected to the upper and lower side of the $i$-th vertebra. The spring force is defined by the Hook's law. The parameter $k_{i j}$ denotes the spring constant. Then the force $\boldsymbol{F}_{i}$ and the torque $\boldsymbol{M}_{i}$ working on the $i$-th vertebrae are expressed by

$$
\begin{gathered}
\boldsymbol{F}_{i}=\boldsymbol{W}_{i}+\sum_{j} \boldsymbol{T}_{i j}, \\
\boldsymbol{M}_{i}=\boldsymbol{r}_{i} \times \boldsymbol{W}_{i}+\sum \boldsymbol{x}_{i j} \times \boldsymbol{T}_{i j},
\end{gathered}
$$

where $\boldsymbol{r}_{i}$ represents the center of mass of the volume, and $\boldsymbol{x}_{i j}$ the vector that points to the contacting point of the spring from the center of mass of the $i$-th vertebra. The symbol ' $X$ ' represents the outer product of two vectors.

As expressed in (21) and (3), $\boldsymbol{F}_{i}$ and $\boldsymbol{M}_{i}$ depend on the positions and the orientations of the $(i-1)$-th and the $(i+1)$-th vertebrae as well as the ones of the $i$-th vertebra. Therefore $\boldsymbol{F}_{i}$ and $\boldsymbol{M}_{i}$ can be expressed by the following equations,

$$
\begin{aligned}
\boldsymbol{F}_{i} & =\boldsymbol{F}_{i}\left(\boldsymbol{r}_{i-1}, \boldsymbol{\theta}_{i-1}, \boldsymbol{r}_{i}, \boldsymbol{\theta}_{i}, \boldsymbol{r}_{i+1}, \boldsymbol{\theta}_{i+1}\right), \\
\boldsymbol{M}_{i} & =\boldsymbol{M}_{i}\left(\boldsymbol{r}_{i-1}, \boldsymbol{\theta}_{i-1}, \boldsymbol{r}_{i}, \boldsymbol{\theta}_{i}, \boldsymbol{r}_{i+1}, \boldsymbol{\theta}_{i+1}\right),
\end{aligned}
$$

where we denote the center of mass of the $i$-th vertebra as $\boldsymbol{r}_{i}$, and the orientation in Euler angle as $\boldsymbol{\theta}_{i} . \boldsymbol{F}_{i}$ and $\boldsymbol{M}_{i}$ must satisfy the following equations because of the equilibrium of the model,

$$
\begin{aligned}
& \boldsymbol{F}_{i}\left(\boldsymbol{r}_{i-1}, \boldsymbol{\theta}_{i-1}, \boldsymbol{r}_{i}, \boldsymbol{\theta}_{i}, \boldsymbol{r}_{i+1}, \boldsymbol{\theta}_{i+1}\right)=\mathbf{0}, \\
& \boldsymbol{M}_{i}\left(\boldsymbol{r}_{i-1}, \boldsymbol{\theta}_{i-1}, \boldsymbol{r}_{i}, \boldsymbol{\theta}_{i}, \boldsymbol{r}_{i+1}, \boldsymbol{\theta}_{i+1}\right)=\mathbf{0} .
\end{aligned}
$$

Therefore, when we denote the number of the vertebrae as $\mathrm{N}$, the positions and the orientations of the $N-2$ vertebrae except the top and the bottom vertebrae are determined by solving the $2 \times(N-2)$ sets of nonlinear equations. These equations can be solved using the Newton-Raphson method.

We assume that the top vertebra of the model supports the weight of the head and arms as expressed in the following equations,

$$
\begin{aligned}
\boldsymbol{F}_{1}=\boldsymbol{W}_{\text {head }}+\boldsymbol{W}_{\text {larm }}+\boldsymbol{W}_{\text {lfarm }}+\boldsymbol{W}_{\text {rarm }}+\boldsymbol{W}_{\text {rfarm }}+\sum_{j} \boldsymbol{T}_{1 j}, \\
\boldsymbol{M}_{1}=\boldsymbol{r}_{\text {head }} \times \boldsymbol{W}_{\text {head }}+\boldsymbol{r}_{\text {larm }} \times \boldsymbol{W}_{\text {larm }}+\boldsymbol{r}_{\text {lfarm }} \times \boldsymbol{W}_{\text {lfarm }} \\
+\boldsymbol{r}_{\text {rarm }} \times \boldsymbol{W}_{\text {rarm }}+\boldsymbol{r}_{\text {rfarm }} \times \boldsymbol{W}_{\text {rfarm }}+\sum_{j} \boldsymbol{x}_{1 j} \times \boldsymbol{T}_{1 j},
\end{aligned}
$$


where $\boldsymbol{W}_{\text {head }}, \boldsymbol{W}_{\text {larm }}, \boldsymbol{W}_{\text {lfarm }}, \boldsymbol{W}_{\text {rarm }}$, and $\boldsymbol{W}_{\text {rfarm }}$ represent the weight of the head and the arms. $\boldsymbol{F}_{1}$ and $\boldsymbol{M}_{1}$ do not satisfy the conditions expressed by (6) and (7) because the position of the top vertebra is determined according to (11). Therefore, we find the top vertebra position which satisfies (6) and (7) by translating the top vertebra along the plumb line that passes through its center of mass.

After the spine posture estimation, we can calculate the forces working on the vertebral body by (2) and (3). In these equations, the terms for the forces of repulsion of the spring should be derived by summing only the forces caused by the springs connected to the upper surface of the vertebral body.

\section{Experimental Results and Discussion}

\subsection{Accuracy of the Model Deformation Method Using MR Images}

To evaluate the accuracy of the spine model deformation, we compared the positions and the orientations of the vertebrae estimated by our method and the ones measured from actual MR images. We assumed that the neck and waist positions were known so that the whole body model fitting to human images was not carried out.

In this experiment, we used three sets of MR images of healthy subject (Fig[3). The datasets $\mathrm{A}$ and $\mathrm{B}$ were the coronal and sagittal images in which a subject was lying straightly on the bed of MRI. The dataset $\mathrm{C}$ was the coronal images in which the subject was lying with his body bending to the right. To construct and determine the initial posture of the spine model, we measured the sagittal diameters, transverse diameters, and the initial positions of the vertebrae from the datasets $\mathrm{A}$ and $\mathrm{B}$. Then the model was deformed to fit it to the neck and waist positions acquired from the dataset $\mathrm{C}$, and we compared the positions and the orientations of the vertebrae resulting from the model fitting with the ones measured from the dataset C. Acquisition parameters of the MR images were: $512 \times 512$ pixels, $0.586 \mathrm{~mm}$ pixel size, 7 slices. The reconstruction pitch was $15 \mathrm{~mm}$ for the image datasets $\mathrm{A}$ and $\mathrm{C}, 8 \mathrm{~mm}$ for the image dataset B. We used the T1-weighted images taken by AIRIS-II (Hitachi Medical Corp.). We employed the Young's modulus of $500 \mathrm{MPa}$ and the Poisson's ratio of 0.3 as the material property of the intervertebral discs [8]. The spring constant $k_{i}$ were determined as the approximations of these values.

We implemented our proposed method on a conventional PC (Pentium 4 $2.8 \mathrm{GHz}$ ). Total computation time was 77.6 seconds. The model deformation was repeated 65 times until the top vertebra reached the neck position obtained from the MR images. Seventy iterations were performed on average to solve the sets of nonlinear equations (6) and (7).

Table 1 shows the comparison results of the positions and orientations of the vertebrae measured from MR images and the ones estimated by our methods. In this table, $\Delta x$ and $\Delta y$ mean the errors along the left-right direction and along the longitudinal direction, respectively. SSD means the sum of squared 


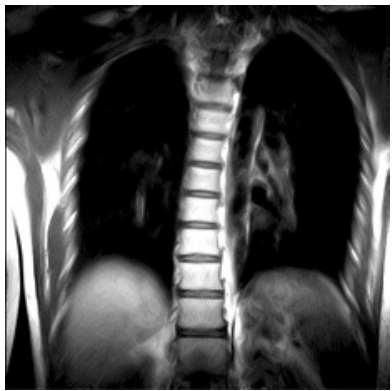

(a)

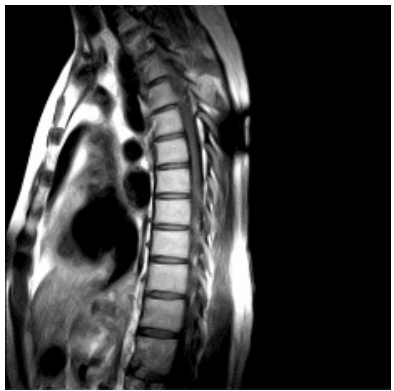

(b)

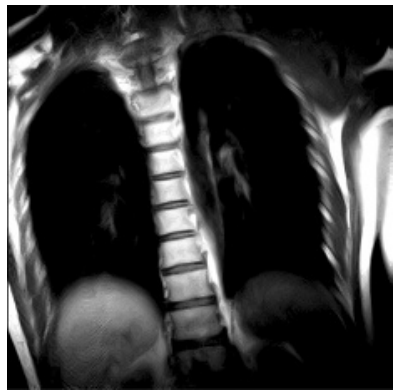

(c)

Fig. 3. Typical slices of each of three datasets A, B, and C of MR images to evaluate accuracy of the spine model deformation. The images (a) and (b) are one of the coronal and sagittal images in the datasets $\mathrm{A}$ and $\mathrm{B}$. The image (c) is one of the coronal images in the dataset $\mathrm{C}$.

differences. The unit of the values is millimeter. The abbreviation ' $\mathrm{T}$ ' and ' $\mathrm{L}$ ' represent thoracic and lumbar vertebrae, respectively.

\subsection{Calculation of the Forces Working on the Lumbar Vertebra 'L5'}

We calculated the physical force on the upper surface of the lumbar vertebra 'L5' by applying the methods to the real human images, and compared the calculation results with the ones performed by Schultz et al. 9. In the Schultz's model which did not consider effect of the dorsal muscles, the physical force on the upper surface of the 'L5' was simply calculated by the sum of the weight of the head, torso, arms, and the weight held by a subject. Schultz solved the equation with respect to the force on 'L5' of the subject holding an upright posture and supporting a weight in the right hand. In our experiment, we assumed the conditions similar to Schultz did. The subject was slowly lifting up the weight of about $4.1 \mathrm{~kg}$ to his elbow height. The weight of the head, torso, and arm of the subject were about $3.6 \mathrm{~kg}, 25.7 \mathrm{~kg}$, and $3.3 \mathrm{~kg}$, respectively. The subject was the same person whose MR images were used in the experiment stated in Sect.4.1. Then the model constructed in the above experiment were used again in this experiment. Fitting the whole human body model to the human images was carried out manually.

Figure 4 shows the examples of the estimated spine postures. The estimated spine postures are overlaid on the figures. The violet ellipsoidal regions represent the whole body model. The computation result of the force working on the lumbar vertebra 'L5' was about $336 \mathrm{~N}$. The experiment was carried out on the same computer resource (Pentium $42.8 \mathrm{GHz}$ ) used in Sect.4.1. Total computation time was about 49 seconds. 
Table 1. The comparison results of the positions and the orientations of the vertebrae measured from MR images and the ones estimated by our methods.

\begin{tabular}{c|ccc|c}
\hline Vertebra & $\Delta x(\mathrm{~mm})$ & $\Delta y(\mathrm{~mm})$ & $\mathrm{SSD}(\mathrm{mm})$ & $\Delta \theta(\mathrm{deg})$ \\
\hline T2 & 9.1 & 1.4 & 9.3 & 12.6 \\
T3 & 11.0 & -4.0 & 11.7 & 4.3 \\
T4 & 13.2 & -1.7 & 13.4 & 2.2 \\
T5 & 11.7 & -1.9 & 11.8 & 2.9 \\
T6 & 10.0 & -1.0 & 10.0 & 0.2 \\
T7 & 8.5 & -1.4 & 8.6 & 0.1 \\
T8 & 7.0 & -0.6 & 7.0 & 1.7 \\
T9 & 4.3 & -0.3 & 4.3 & 3.1 \\
T10 & 1.2 & 0.2 & 1.2 & 6.0 \\
T11 & 0.1 & 1.7 & 1.7 & 4.1 \\
T12 & -1.4 & 0.9 & 1.6 & 2.4 \\
L1 & 0.1 & 3.2 & 3.2 & 3.3 \\
L2 & 0.4 & 2.7 & 2.7 & 0.9 \\
L3 & 1.1 & 3.4 & 3.5 & 1.1 \\
L4 & 1.7 & 4.3 & 4.6 & 1.5 \\
\hline M $\pm \sigma$ & $5.2 \pm 4.9$ & $0.5 \pm 2.2$ & $6.3 \pm 6.0$ & $3.1 \pm 3.0$ \\
\hline
\end{tabular}

\subsection{Discussion}

As shown in Table 1, the average of the positional and rotational variation of the vertebrae were $6.3 \mathrm{~mm}$ and 3.1 degrees, respectively. According to our measurement of the position of the vertebra 'T4' based on the MR images, it moved by $68.3 \mathrm{~mm}$ from its original position when the subject bent his body. The proposed method estimated its position by $13.4 \mathrm{~mm}$ of error. Therefore, the error of the estimation of ' $\mathrm{T} 4$ ' is about $20 \%$. The computation result of the force working on the lumbar vertebra 'L5' was about $336 \mathrm{~N}$, while the force was about $390 \mathrm{~N}$ in the Schultz's simple model which did not have the dorsal muscles [9]. Therefore, it turned out that our model can estimate the physical forces to the vertebrae as accurately as the forces estimated by the Schultz's method.

Compared with the Schultz's and others' methods, the main advantage of the proposed method is that we can understand the state of the spine with respect to real human motions the subject actually takes. The previously proposed methods did not allow such physical force computation based on real postures as our method did. Because the proposed method estimates physical forces from the human images, the proposed method helps us to determine the initial and boundary conditions necessary for precise physical force analyses such as finite element methods.

The dorsal muscles are very important tissues in terms of the forces to the vertebrae. Some experimental results reported by other researchers indicate that more than $1000 \mathrm{~N}$ of the forces act on the lumbar vertebrae due to a function of the dorsal muscles. We do not model the dorsal muscles since it is difficult to determine the muscle forces. However, to calculate the forces more precisely, we have to consider the interaction between the dorsal muscles and the vertebrae. 


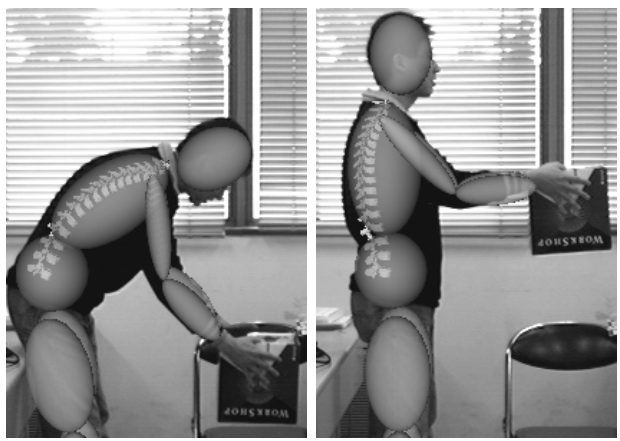

Fig. 4. Examples of the estimated spine postures.

\section{Conclusion}

In this paper, we have proposed a spine model and a method for estimating the spine posture from human images taken by video cameras. According to the preliminary experiments based on one real MR image data set of only one subject person, the methods estimated the positions of the vertebrae within positional shifts of about $6.3 \mathrm{~mm}$ and the rotational variation of about 3.1 degrees. We also confirmed that the methods calculated the reasonable approximation of the physical forces working on the vertebral body. Future work includes: (1) validation of accuracy of the model deformation method using a large set of MR image sets, (2) application to a large set of human images and the discussion about the method for evaluating the estimated spine posture, and (3) precise validation of the calculated physical forces on the vertebra from the viewpoint of anatomy and biomechanics.

Acknowledgements. Authors wish to thank Dr. Hiroshi Iseki and Dr. Kiyoshi Naemura of Tokyo Women's Medical University for cooperating with them to take the MRI images. This study was partly supported by the Grants-in-Aid for Scientific Research and the 21st Century COE Program from Japan Society for the Promotion of Science, Grants-in-Aid for Cancer Research from the Ministry of Health, Labor and Welfare of Japan.

\section{References}

1. Chen, C.S. et al.: Stress analysis of the disc adjacent to interbody fusion in lumbar spine. Medical Engineering \& Physics. 23 (2001) 483-491

2. Nabhani, F. et al.: Computer modeling and stress analysis of the lumbar spine. J. Materials Processing Technology. 127 (2002) 40-47

3. Pitzen, T. et al.: A finite element model for predicting the biomechanical behaviour of the human lumbar spine. Control Engineering Practice. 10 (2002) 83-90 
4. Monheit, G. and Badler, N.I.: A Kinematic Model of the Human Spine and Torso. IEEE Computer Graphics and Applications. (1991) 29-38

5. Otake, Y. et al.: Development of 4-Dimensional Human Model System for the Patient after Total Hip Arthroplasty. Medical Image Computing and ComputerAssisted Intervention. (2002) 241-247

6. Furukawa, D. et al.: Human Spine Posture Estimation from 2D Frontal and Lateral Views Using 3D Physically Accurate Spine Model. IEICE Transactions on Information and Systems. E87-D (2004) 146-154

7. National Library of Medicine: The Visible Human Project. http://www.nlm.nih.gov/research/visible/visible_human.html

8. Kumaresan, S. et al.: Finite element modeling of the cervical spine: role of intervertebral disc under axial and eccentric loads. Medical Engineering \& Physics. 21 (1999) 689-700

9. Schultz, A.B. and Andersson, B.J.: Analysis of Loads on the Lumbar Spine. Spine. 6 (1981) 76-82

10. Bregler, C. and Malik, J.: Tracking People with Twists and Exponential Maps. Int. Conf. Computer Vision and Pattern Recognition. (1998) 8-15 